\title{
Is ultra low-dose CT with tin filtration useful for examination of SI joints? Can it replace $X$-ray in diagnostics of sacroiliitis?
}

\author{
Eva Korcakova ${ }^{\mathrm{a}, \mathrm{b}}$, Jana Stepankova ${ }^{\mathrm{c}}$, David Suchy ${ }^{\mathrm{d}}$, Petr Hosek ${ }^{\mathrm{b}}$, Kristyna Bajcurova ${ }^{\mathrm{a}, \mathrm{b}}$, Jan Pernicky ${ }^{\mathrm{a}}$, Hynek Mirka ${ }^{\mathrm{a}, \mathrm{b}}$
}

\begin{abstract}
Objectives. The first objective of our study was to determine the radiation exposure received by patients during tinfiltrated ultra-low-dose computed tomography (TFULDCT) of sacroiliac joints and to compare those to conventional $\mathrm{X}$-ray doses. For comparison, we added a cohort examined by low-dose CT (LDCT) without tin filtration. The second objective was to compare the results of TFULDCT and X-ray in the detection of sacroiliitis.

Methods. Our retrospective study covered 45 patients, who were examined for suspected axial spondyloarthritis $(\mathrm{AxSpA})$. The first group underwent TFULDCT as well as conventional radiography (CR); the second group underwent LDCT only without tin filtration. Effective doses of TFULDCT, LDCT and CR were calculated by an experienced medical physicist. TFULDCT and CR were independently evaluated by three investigators, who decided on the presence or absence of rheumatoid inflammatory bone changes. The results were statistically evaluated.

Results. In our cohort, the median effective dose for TFULDCT was $0.11 \mathrm{mSv}$, range (0.06-0.40 mSv), for LDCT $0.5 \mathrm{mSv}$ (0.29-0.89 mSv), and for CR $0.25 \mathrm{mSv}(0.06-1.87 \mathrm{mSv})$. We proved that TFULDCT produces a significantly lower percentage of uncertain results (23.3\%; $95 \% \mathrm{Cl}: 11.3-41.6 \%)$ than CR (66.7\%; $95 \% \mathrm{Cl}: 48.3-81.1 \%)$.

Conclusions. Tin filtration helps to reduce $\mathrm{CT}$ radiation exposure to values lower than those resulting from CR. TFULDCT offers better overall diagnostic performance than CR. Our results prove that TFULDCT can replace CR in the diagnosis of sacroiliitis in the radiographical stage of AxSpA.
\end{abstract}

Key words: radiation, computed tomography, tin filtration, sacroiliitis, axial spondyloarthritis

Received: August 2, 2020; Revised: December 28, 2020; Accepted: January 15, 2021; Available online: February 4, 2021 https://doi.org/10.5507/bp.2021.008

(c) 2022 The Authors; https://creativecommons.org/licenses/by/4.0/

${ }^{a}$ Department of Imaging methods, University hospital in Plzen, Czech Republic

${ }^{b}$ Biomedical Center, Faculty of Medicine in Pilsen, Charles University, Czech Republic

'Division of Medical physics, University hospital in Plzen, Czech Republic

${ }^{d}$ Clinical Rheumatology, University hospital in Plzen, Czech Republic

Corresponding author: Eva Korcakova, e-mail: korcakovae@fnplzen.cz

\section{INTRODUCTION}

Imaging methods play a crucial role in the diagnosis of sacroiliitis in patients suffering from axial spondyloarthritis (AxSpA). Magnetic resonance imaging (MRI), which is currently considered to be the gold standard in $\mathrm{AxSpA}$ diagnostics, allows the detection of bone marrow oedema occurring in the early phase of the disease. The period of the disease in which bone marrow oedema and inflammatory changes on soft tissues are positive but there are no signs of bone changes on conventional radiography (CR) is called the non-radiographic stage of AxSpA. MRI has also been used to detect bone change activity ${ }^{1}$. Zero radiation is advantageous, especially for young patients. MRI is able to detect bone changes; however, if these changes are minor in the beginning - especially small bone bridges and joint space irregularities without the presence of bone oedema in close proximity - it could lead to diagnostic problems ${ }^{2}$.

The most commonly used method suitable for the detection of bone changes, and hence it is known as the radiological stage of the disease, is $\mathrm{CR}$, which is easily available and inexpensive but provides only low sensitivity for the detection of minor bone changes. The detection of bone changes in CR lags behind the clinical signs by several years ${ }^{3,4}$. Computed tomography (CT) has the best capability to display minor bone changes; however, it is unable to assess bone marrow oedema ${ }^{5}$. CT is useful for detecting minor bone changes and, therefore, proves the transition between the non-radiological and radiological stages of the disease earlier than X-ray. From a clinical point of view, it is suitable for the early detection of ankylosing spondylitis during the period when bone changes are still minor and difficult to detect on CR. Nevertheless, CT has not been widely used due to the high exposure to radiation ${ }^{6,7}$.

Recent developments in CT technology have allowed for a significant dose reduction ${ }^{8-10}$. This is possible thanks to automatic dose optimization systems, iterative reconstruction, and spectral shaping by tin filtration ${ }^{11}$. The use of tin filtration modifies the spectrum and exposure to radiation is reduced by blocking low-energy photons. Spectra with tin filtration are more dose efficient than traditional spectra (less noise) ( ref. $^{12,13}$ ); tin filtration is recommended for the non-contrast imaging of high-contrast objects ${ }^{13-15}$. Although tin filtration is recommended 
for bone examinations, no work has yet been published that focuses on the use of tin filtration in the examination of sacroiliac (SI) joints.

The objective of our study was to assess the radiation exposure necessary to examine SI joints. We compared effective doses from tin-filtrated ultra-low-dose computed tomography (TFULDCT) with effective doses from lowdose computed tomography (LDCT) without tin filtration and from X-ray. We further compared the diagnostic performance of TFULDCT and X-ray in the detection of bone changes (Fig. 1).

\section{METHODS}

\section{Patients}

Our retrospective study evaluated a study group of 30 consecutive patients who underwent both TFULDCT of SI joints and CR of pelvis or SI joints. A reference group of 15 subjects was examined by LDCT without tin filtration. We did not examine X-ray doses in this group. All patients were referred from a rheumatology centre with suspected radiographic stage AxSpA and all had suffered from lower back pain for at least one year. BMI, HLA B27, C-reactive protein levels, and response to non-steroid antiinflammatory drugs (NSAIDs) were recorded for each patient in the study group ${ }^{16}$.

Patients were informed of the risk of radiation and signed an informed consent from. The study was approved by the local ethics committee.

\section{Examination}

CT examinations were performed with a multidetector scanner Somatom Definition Drive (Siemens, Germany). The study group was examined with a low-dose protocol with a following definition: tube voltage $100 \mathrm{kV}$, automatic modulation of the tube current (Care dose 4D) with reference value $95 \mathrm{mAs}$, tin filtration thickness of $0.4 \mathrm{~mm}$, collimation $0.6 \mathrm{~mm}$, pitch 0.8 . The ADMIRE reconstruction algorithm was used. The examinations were performed without contrast media administration. The scanning range was limited to only the SI joint region.

The reference group was examined on the same scanner, as well as the same scanning protocol, except the tin filtration.

CR was done in the standard way according to the practices of the performing institutions. Either one anteroposterior (AP) projection or two oblique projections of SI joints were available.

\section{Evaluation}

CR and CT data sets were evaluated, and dosage calculations were performed by an experienced medical physicist using ImpactDose 2.3, patient model - real patient data (CT Imaging GmbH, Germany), and PCXMC 2.0 (X-ray, STUK Finland). The estimate of the dose was determined for each frame using the following parameters: displayed dose-area product $\left(\mathrm{mGy} . \mathrm{cm}^{2}\right)$, applied voltage $(\mathrm{kV})$, used filtration, radiation field size $(\mathrm{cm})$, projection type, patient anatomical parameters. An effective dose (expressed in $\mathrm{mSv}$ ) was used for the analysis. Other values only calculable for CT examinations were recorded.

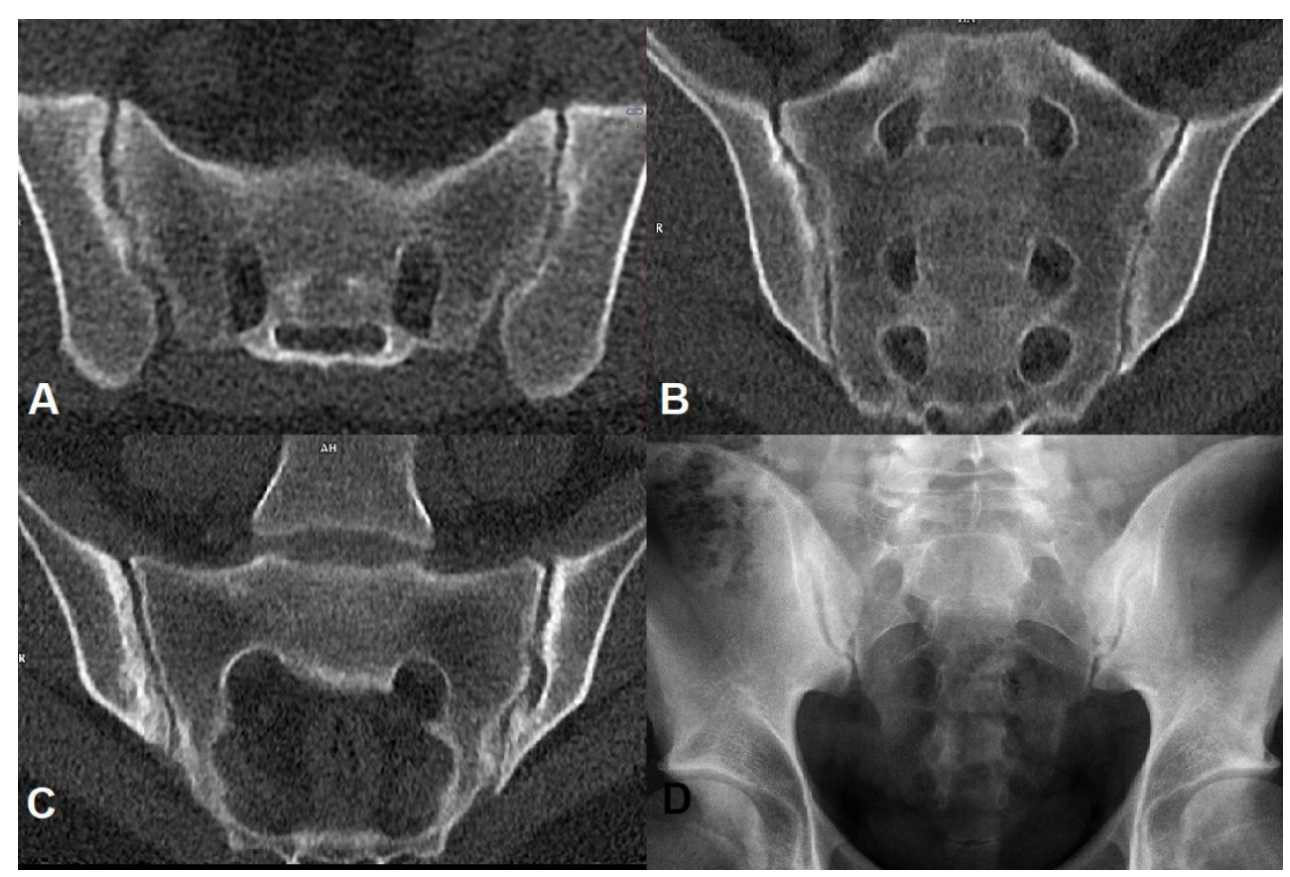

Fig. 1. Man, 29 years old, suffering from long-term low back pain, HLA B27 negative, CRP $10 \mathrm{mg} / \mathrm{L}$, BMI 25. TFULDCT in axial (A), oblique (B), and coronal (C) plane, effective radiation dose 0.12 $\mathrm{mSv}$. In all pictures are seen small usurations, the joint cavities are irregular, locally widespread and light subchondral sclerosis is present. Any bone bridges are evident. We evaluated this finding as grade two according to the New York criteria. X-ray (D) of pelvis, effective radiation dose 0.25 $\mathrm{mSv}$. Bone changes on SIJ are uncertain, the joint space irregularity is more pronounced on left side, despite the changes are more expressed on right side in the TFULDCT images. 
All CR and CT scans of the study group were evaluated by three radiologists with a minimum of 5 years of experience using the Syngovia (Siemens, Germany) diagnostics workstation. Datasets were evaluated in the axial and coronal planes; if necessary, reconstruction in other planes were added. Evaluators worked independently and oblivious to other clinical findings or the results of the other method (with a time gap of at least two weeks between methods). Each finding was classified according to modified New York classification (Table 1). The values from the three radiologists were averaged (arithmetic mean) to obtain one final CT score and one final CR score for each patient. This score was then evaluated quantitatively, as well as categorically, after classifying values greater than 3 as positive, values smaller than 1 as negative, and values between 1 and 3 (including threshold values) as uncertain/indecisive.

The results were evaluated with respect to a reference binary finding (positive/negative) obtained as the consensual decision of a senior radiologist specialized in the field of rheumatic diseases and a senior rheumatologist. Both experts were familiar with the complete medical documentation of the patients. The decision was made on the basis of the evaluation of complete imaging and clinical documentation, clinical findings, and the results of laboratory tests. In the case of a positive finding, the experts classified it according to the modified New York classification as stage 2-4.

During the evaluation, the quality of the documentation was monitored and the evaluators decided whether the image quality was sufficient or insufficient. The evaluation of image quality was also applied to the reference group.

\section{Statistics}

$\mathrm{CT}$ and $\mathrm{CR}$ doses were compared using the Wilcoxon matched pairs test. The relationship between the effective dose and BMI were independently analysed for both methods using Spearman's correlation (continuously) and the Mann-Whitney U test (after stratification according to BMI). Even though non-parametric methods were preferred due to mostly non-normal variable distribution, the average sacroiliitis assessment scores were examined using repeated measures ANOVA (with the factor of 'reference patient status' at two measurement levels according to 'method', i.e. CT or X-ray), in order to better reflect the study design and investigate the factors' interaction. After producing the final CT and X-ray findings by classifying the scores, the diagnostic utility of both methods was evaluated by Fischer's exact test. Confidence intervals of percentages were calculated according to Agresti and Coul1 ${ }^{17}$. All reported $P$-values are two-tailed and the level of statistical significance was set at $\alpha=0.05$. Statistical processing and testing were performed in STATISTICA data analysis software system (Version 12; StatSoft, Inc, 2013; www.statsoft.com).

\section{RESULTS}

\section{Patients}

Study population included 14 men, 16 women, age median 43.5 years (range 28-61 years). Median body mass index (BMI) was $27.7 \mathrm{~kg} / \mathrm{m}^{2}\left(20.9-38.8 \mathrm{~kg} / \mathrm{m}^{2}\right)$. The reference group included 6 men and 9 women, age median 48 years (range $38-68$ years). Median BMI $28.1 \mathrm{~kg} / \mathrm{m}^{2}$ $\left(19.5-43.4 \mathrm{~kg} / \mathrm{m}^{2}\right)$.

On the basis of the consensual decision of both experts there were 16 patients in our study cohort sacroiliitis-positive. This means that the findings were consistent with typical bone changes in sacroiliitis. According to the New York classification, ten cases were stage 2, five stage 3, and one stage 4. Further details in Table 2.

Table 1. Evaluation according the New York classification.

\begin{tabular}{|c|c|c|c|c|c|}
\hline Score & 0 & 1 & 2 & 3 & 4 \\
\hline Result & negative & $\begin{array}{l}\text { indecisive but } \\
\text { propably negative }\end{array}$ & indecisive & $\begin{array}{l}\text { indecisive but } \\
\text { propably positive }\end{array}$ & positive \\
\hline $\begin{array}{l}\text { New York } \\
\text { classification }\end{array}$ & NY 0 & NY 1 & NY 1 & NY 1 & NY 2,3,4 \\
\hline
\end{tabular}

Table 2. Classification of our cohort.

\begin{tabular}{lcc}
\hline Result & Sacroiliitis positive & Sacroiliitis negative \\
\hline Number & 16 & 14 \\
HLA B27 positive & 11 & 4 \\
NSAIDs positive reaction & 10 & 4 \\
CRP elevated (over 8 mg/L ) & 9 & 1 \\
Average level of CRP & $13.7 \mathrm{mg} / \mathrm{L}(3-46 \mathrm{mg} / \mathrm{L})$ & $3.3 \mathrm{mg} / \mathrm{L}(0-21 \mathrm{mg} / \mathrm{L})$ \\
Log-time low back pain & 16 & 14 \\
\hline
\end{tabular}

\footnotetext{
*Decision about split into two group was done on the basis of two experts agreement
} 
Table 3. Doses for each method.

\begin{tabular}{lcccc}
\hline & Effective dose $(\mathrm{mSv})$ & CTDI $_{\text {vol.32 }}(\mathrm{mGy})$ & DLP $\left(\mathrm{mGy}^{*} \mathrm{~cm}\right)$ & Scan length $(\mathrm{cm})$ \\
\hline TFULDCT & $0.11(0.06-0.40)$ & $0.62(0.39-3.50)$ & $11.58(6.83-19.60)$ & $9.74(5.90-86.55)$ \\
LDCT & $0.50(0.29-0.89)$ & $3.11(1.54-7.26)$ & $45.28(20.98-122.93)$ & $10.77(8.07-13.89)$ \\
CR & $0.25(0.06-1.87)$ & & & \\
\hline
\end{tabular}

CTDI $_{\text {vol.32 }}$, volumometric CT dose index; DLP, dose length product; TFULDCT, tin filtrated ultra-low-dose computed tomography; LDCT, lowdose computed tomography; $\mathrm{CR}$, conventional radiography (X-ray)

Median values are cited.

\section{Radiation dose}

The median effective dose for TFULDCT was 0.11 $\mathrm{mSv}$, range (0.06-0.40 mSv) while the median effective dose for CR was $0.25 \mathrm{mSv}(0.06-1.87 \mathrm{mSv})$. In the reference LDCT group the median effective dose was 0.50 $\mathrm{mSv}(0.29-0.89 \mathrm{mSv})$. The complete results can be seen in Table 3. Twenty-five patients $(83 \%)$ had undergone two oblique projections focused on an SI joint, only five (17\%) had had one AP projection of the whole pelvis. Doses from TFULDCT were thus significantly lower than CR and LDCT doses (Wilcoxon matched pairs test, $P$ 0.001). Both TFULDCT- and radiograph-associated doses significantly positively correlated to patient BMI (Spearman correlation, $P<0.001$ and $P=0.048$, respectively). The relationship was considerably stronger for TFULDCT (Spearman's $\rho=0.701)$ in comparison to CR $(\rho=0.363)$ (Fig. 2).

The same was shown after stratifying the patients in two groups according to BMI. The group with a BMI lower than 30 received a median TFULDCT effective dose of $0.10 \mathrm{mSv}(0.06-0.16 \mathrm{mSv})$ and a median CR dose of $0.24 \mathrm{mSv}(0.06-1.16 \mathrm{mSv})$. In the group with a BMI of 30 and above, the TFULDCT dose median was $0.18 \mathrm{mSv}$ (0.06-0.40 mSv), while for the CR dose it was $0.39 \mathrm{mSv}$ $(0.2-1.87 \mathrm{mSv})$. The reference group examined without

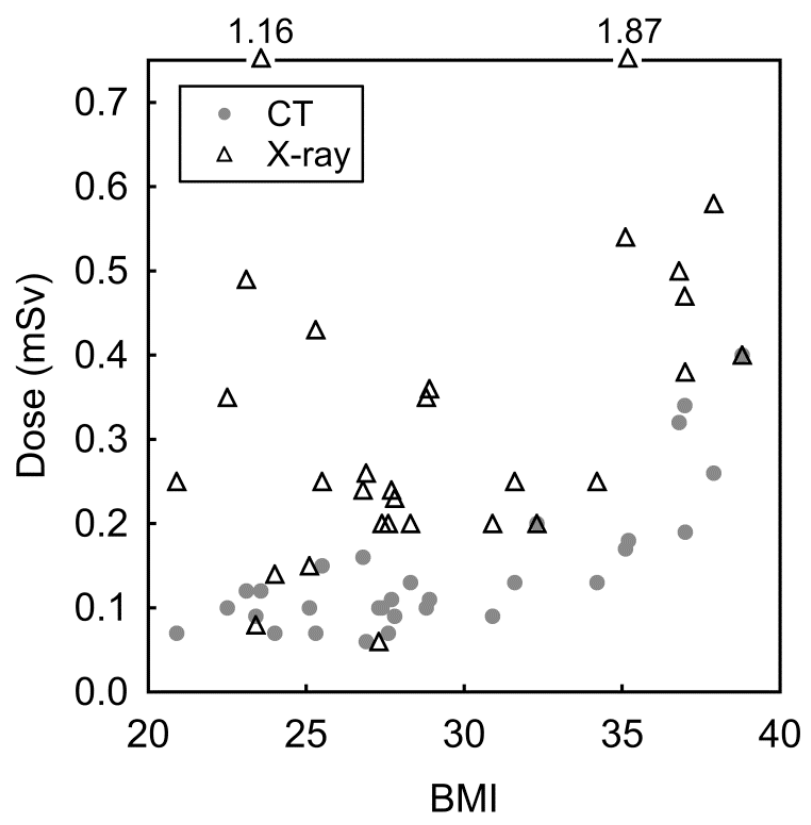

Fig. 2. The dependence radiation dose on BMI. Both TFULDCT- and radiograph-associated doses were significantly positively correlated to patients' BMI. tin filtration was not analysed in more detail due to the small number of patients.

\section{Evaluation of diagnostic performance}

When the average sacroiliitis assessment scores were analysed, TFULDCT showed a significantly bigger difference between the scores reported for positive and negative patients than CR (i.e. significant interaction between the factors of method and reference patient status, $P<0.001$ ). TFULDCT thus showed a better capability to discriminate positive and negative patients (Fig. 3). We observed no substantial difference in the overall mean scores provided by TFULDCT and CR, indicating no evidence of systematic mutual bias between the two methods, i.e. no global tendency of one method to report higher/lower values than the other (effect of the method factor, $P=0.435$ ).

After classification of the collected scores as positive/indecisive/negative, TFULDCT provided a decisive result in 23 out of 30 cases $(76.7 \%)$, thus achieving a significantly higher percentage of decisive findings than CR (Fisher's exact test, $P=0.002$ ), which offered a definitive decision for only 10 patients $(33.3 \%)$. All of the 23 decisive TFULDCT findings were correct with respect to the reference (12 true negative and 11 true positive), while CR provided 9 true results ( 5 true negative and 4

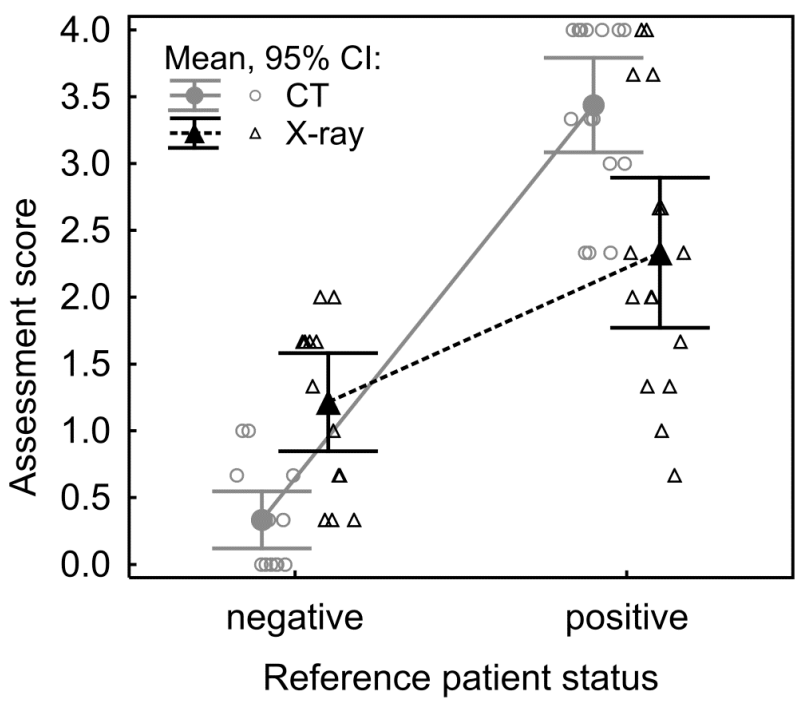

Fig. 3. The assessment score for positive and negative patients in CT and X-ray. TFULDCT showed better ability to discriminate between positive and negative results. 


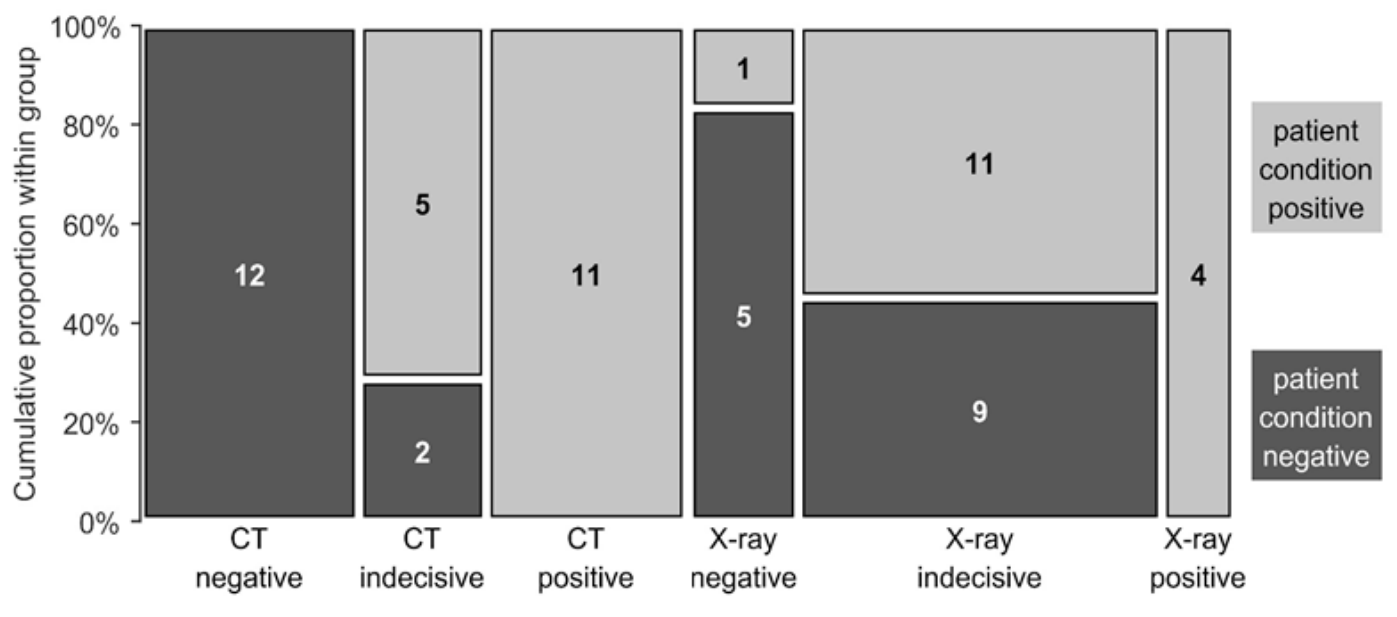

Fig. 4. Overall results of TFULDCT and X-ray.

true positive) and 1 false negative finding (Fig. 4). When comparing the proportions of diagnostically useful, i.e. definite and true findings, TFULDCT show significantly better overall diagnostic performance ( 23 out of 30 ) than CR ( 9 out of 30; Fisher's exact test, $P<0.001$ ).

\section{Quality of images}

The quality of the available images was assessed as sufficient for evaluation in all cases.

\section{DISCUSSION}

We performed a retrospective study comparing the radiation exposure and diagnostic performance of TFULDCT and CR in the diagnostics of sacroiliitis. The assessment of the effective dose was used for dose-comparison as it is a parameter calculable for both CT and $\mathrm{CR}$ and best reflects the amount of radiation received by the patient. Various parameters are used to indicate radiation exposure in $\mathrm{CT}$. $\mathrm{CTDI}_{\mathrm{vol}}$ indicates the radiation output relative to the PMMA phantom, not a patient's real dose. This parameter can be converted to the size-specific dose estimation (SSDE) parameter, which recalculates the CTDI ${ }_{\mathrm{vol}}$ on the patient's body area using a conversion factor (in relation to the LAT and AP dimensions of the patient) (ref. ${ }^{18-21}$ ). These parameters can be easily obtained from the CT protocol, but they cannot be used to compare doses between CT and CR. We used these parameters uniquely for the comparison of TFULDCT with LDCT. BMI and effective diameter (the LAT and AP dimensions of the patient) provide information about the type of stature, which is important to consider because a higher volume body requires a higher dose, as our results also confirmed.

The dose reduction is influenced not only by the chosen low-dose protocol, but also by the use of tin filtration. This was proven by the comparison with the data from the reference group, which was not investigated further and was evaluated only to demonstrate the effect of tin filtration on the level of radiation exposure. As can be seen from our results, tin filtration effectively reduces the dose of LDCT.

The effective dose produced by TFULDCT was low in our cohort, almost as low as half of that of the CR. The low radiation allows more frequent use of TFULDCT in patients with suspected AxSpA. Standard CR in our cohort consisted of two projections of SI joints, which could explain the higher effective dose. The constitution of a patient's body significantly influences the dose for both methods; this was reflected in our observation as a positive correlation of BMI with both effective doses. This dependence was considerably stronger for CT. This may be explained by the automatic modulation of tube current used during the TFULDCT examination. While the higher volume of a patient's body naturally absorbs more radiation in both TFULDCT and CR, the modulation algorithm in TFULDCT probably also dictated higher tube current values (and therefore higher radiation intensity) for high-BMI patients in order to achieve the required image quality.

In literature, we have found three studies focusing on LDCT of SI joints. In the Canadian study the CT effective doses for SI joints were calculated from abdominal LDCT and were compared with doses of CR for pelvis or SI joints. The mean calculated dose in this study was $0.42 \mathrm{mSv}$ for CT and $0.15 \mathrm{mSv}$ for CR (ref. ${ }^{22}$ ). There are several differences between ours and the Canadian study. Firstly, doses for the SI joint regions were calculated from an abdominal CT, while we declared the real doses for the targeted examination of the SI joint regions. Secondly, the examinations were performed on a different CT device and with different exposure parameters. Thirdly, tin filtration was not used in the Canadian study. It is therefore not fully accurate to compare the CT doses between ours and the above mentioned study; CR doses were slightly lower than those we observed in our study.

The second study which used LDCT for the evaluation of bone changes in sacroiliitis was published by German authors. This work compared the results of LDCT, MR and CR in the detection of bone changes in sacroiliitis. LDCT was used as the gold standard in this study. LDCT 
doses were $0.5 \mathrm{mSv}$, maximum $1.4 \mathrm{mSv}$. These results are better comparable to our results because this study was performed on a similar CT device and the exposure parameters were similar to ours. Tin filtration was not used. The doses of LDCT published in this study are comparable to the doses in our group examined without tin filtration ${ }^{2}$.

The third study, published in 2002, compared effective doses of semi-coronal CT, axial CT, and CR. Their results showed that the lowest dose was produced with semi-coronal CT, and that the dose was six times lower than that of axial CT. Unlike our study, measurements were performed on an anthropomorphic model. This means that absorption differences in bodies of different constitutions were not taken into account. The scanning parameters used were also different. They examined in 5 $\mathrm{mm}$ sections, obtaining a logically lower resolution than sub-millimetre sections and not allowing for multi-planar reconstructions without loss of resolution. Tin filtration was not used. The effective doses reported in this publication were similar to our results ${ }^{23}$.

Some published studies used LDCT in the detection of bone changes in the spine in patients suffering from $\operatorname{AxSpA}$, but without using tin filtration ${ }^{8-10}$.

Our results confirmed that TFULDCT evaluates bone changes with a high level of certainty and with a low percentage of unclear results, and all the definite results were correct. On the contrary, in CR most of the results are ambivalent.

Similar results were reported by a French study from $2012\left(\right.$ ref. $\left.^{6}\right)$. They showed excellent inter-reader reliability on standard-dose CT, and moderate on CR. CT was also more accurate in the detection of sacroiliitis and the staging of the disease, especially for stages 2 and 3. Several studies used CT as a reference method to assess the effectivity of MRI or CR. A Danish study comparing CT and MRI showed that $\mathrm{CT}$ has a higher sensitivity to assess changes in the width of the joint space, and to detect bone formations at entheses where MRI had failed. In contrast, MRI had a higher sensitivity to detect bone oedema and fat deposition. Both methods had similar results in the detection of bone erosion and sclerosis ${ }^{24}$. The aforementioned study from Germany demonstrated that CR missed more than half of the structural bone changes on SI joints compared to LDCT. In contrast, the results of MRI in evaluating bone changes were comparable to LDCT. The only exception was sclerosis, where CR had better results than MRI (ref. ${ }^{2}$ ).

A disadvantage of CT is a longer evaluation time, as well as higher costs in comparison to CR. Aside from these shortcomings in CT, our study has conclusively shown the benefits of TFULDCT. The relatively small number of included patients could be seen as a minor limitation.

Based on our results, it is possible to recommend replacing CR with the more informative LDCT for diagnosing sacroiliitis. The very low level of radiation exposure supports TFULDCT as the preferred method in the diagnostics of radiographic form AxSpA. Further investi- gation will be necessary to assess whether CT is able to move the boundaries of radiological form of $\mathrm{AxSpA}$ to earlier stages.

\section{ABBREVIATIONS}

TFULDCT, Tin filtrated ultra-low-dose computed tomography; LDCT, Low-dose computed tomography; SI, Sacroiliac; CR, Conventional radiography (X-ray); AxSpA, Axial spondyloarthritis.

Acknowledgement: This article was supported by the project of the Ministry of Health-Conceptual Development of Research Institutions 00669806 - FN Plzen, by the project of the Charles University Prague Progress Q36, by the grant UNCE/MED/006 Charles University Center of clinical and experimental liver surgery, and by the European Regional Development Fund - Project „Application of Modern Technologies in Medicine and Industry" (No. CZ.02.1.01/0.0/0.0/17_048/0007280).

Author contributions: EK: manuscript writing, literature search, data analysis; JS: data analysis, manuscript writing; DS, HM: data analysis, final approval; PH: statistical analysis, manuscript writing; KB, JP: data analysis

Conflict of interest statement: None declared.

\section{REFERENCES}

1. Lambert RG, Bakker PA, van der Heijde D, Weber U, Rudwaleit M, Hermann KGA, Sieper J, Baraliakos X, Bennett A, Braun J, BurgosVargas R, Dougados M, Pedersen SJ, Jurik AG, Maksymowych WP, Marzo-Ortega H, Ostergaad M, Poddubnyy D, Reijnierse $M$, van den Bosch F, van der Horst-Bruinsma I, Landewé R. Defining active sacroiliitis on MRI for classification of axial spondyloarthritis: update by the ASAS MRI working group. Ann Rheum Dis 2016;0:1-6.

2. DiekhoffT, Hermann KG, Greese J, Schwenke C, Poddubnyy D, Hamm $\mathrm{B}$, Sieper J. Comparison of MRI with radiography for detecting structural lesions of the sacroiliac joint using CT as standard of reference: results from the SIMACT study. Ann Rheum Dis 2017;76:1502-8.

3. Sykes MP, Doll H, Senqupta R, Gaffney K. Delay to diagnosis in axial spondyloarthritis: are we improving in the UK? Rheumatology 2015;54:2283-84.

4. Pavelka K. Early diagnosis of ankylosing spondylitis. Vnitr Lek 2006; 52(7-8):726-9.

5. Slobodin G, Hussein H, Rosner I, Eshed I. Sacroiliitis - early diagnosis is key. J Inflamm Res 2018;11:339-44.

6. Devauchelle-Pensec V, D’Agostino MA, Marion J, Lapier M, JousseJoulin S, Colin D, Chary-Vackenaere I, Loeuille ChM, Aegerter P, Guis S, Gaudin P, Breban M, Saraux A.. Computed tomography scanning facilitates the diagnosis of sacroiliitis in patients with suspected spondylarthritis. Arthritis Rheum 2012;64:1412-27.

7. Geijer M, Göthlin GG, Göthlin JH. The clinical utility of computed tomography compared to conventional radiography in diagnosing sacroiliitis. A retrospective study on 910 patients and literature review. J Rheumatol 2007;34:1561-65.

8. Maksymowych W, Lambert R. Low-dose CT for spondyloarthritis - a brilliant new chapter? Nat Rev Rheumatol 2018;14:130-31.

9. de Bruin F, de Koning A, van den Berg R, Baraliakos X, Braun J, Ramiro $S$, van Gaalen F, Reijnierse M, van der Heijde D. Development of the CT Syndesmophyte Score (CTSS) in patients with ankylosing spondylitis: data from the SIAS cohort. Ann Rheum Dis 2018;77(3):371-77. doi:10.1136/annrheumdis-2017-212553

10. de Koning A, de Bruin F, van den Berg R, Ramiro S, Baraliakos X, Braun $J$, van Gaalen FA, Reijnierse M,van der Heijde D. Low-dose CT detects more progression of bone formation in comparison to conventional 
radiography in patients with ankylosing spondylitis: results from the SIAS cohort. Ann Rheum Dis 2018;77:293-99.

11. Rego, S, Yu, L, Bruesewitz, M, Vrieze, T, Kofler, J, McCollough, C. CARE Dose4D CT Automatic Exposure Control System: Physics Principles and Practical Hints. Radiological Society of North America 2007 Scientific Assembly and Annual Meeting, November 25 - November 30, 2007, Chicago IL. http://archive.rsna.org/2007/5011233.html

12. Haubenreisser $H$, Meyer M, Sudarski S, Allmendinger T, Schoenberg SO, Henzler T. Unenhanced third-generation dual-source chest CT using a tin filter for spectral shaping at $100 \mathrm{kVp}$. Eur J Radio 2015;84:1608-13.

13. White paper. Shaping the beam. Versatile filtration for unique diagnostic potential within Siemens Healthineers CT. Mark Woods, Marcus Brehm. Available from: https://www.siemens-healthineers. com/computed-tomography/technologies-and-innovations/tinfilter (accessed on July 10, 2020)

14. Lell MM, May MS, Brand M, Eller A, Buder T, Hofmann E, Uder M, Wuest W. Imaging the Parasinus Region with a Third-Generation Dual-Source CT and the Effect of Tin Filtration on Image Quality and Radiation Dose. AJNR Am J Neuroradiol 2015;36(7):1225-30. doi:10.3174/ajnr.A4270

15. Martini K, Higashigaito K, Barth BK, Baumueller S, Alkadhi H, Frauenfelder T. Ultralow-dose CT with tin filtration for detection of solid and sub solid pulmonary nodules: a phantom study. $\mathrm{Br} J$ Radiol 2015;88:20150389.
16. https://www.smartbmicalculator.com/

17. Agresti, A, and Coull, BA. Approximate is Better than "exact" for interval estimation of binomial proportions, The American Statistician 1998:52:119-26.

18. Brink JA, Morin RL. Size-specific dose estimation for CT: How should it be used and what does it mean? Radiology 2012;265:666-68.

19. Sukupova L. What does CTDIvol mean and is this parameter suitable for the determination of patient's doses? Ces Radiol 2015;69(3):194200.

20. Seiber JA, Boone JM, Wootton-Gorges SL, Lamba R. Dose is not always what it seems: where very misleading values can result from volume CT dose index and dose length product. J Am Coll Radiol 2014;11(3):233-37.

21. Lee $\mathrm{Ch}$. How to estimate effective dose for $\mathrm{CT}$ patients. Eur Radiol 2020;30:1825-27.

22. Chahal BS, Kwan AL, Dillon SS, Olubaniyi BO, Jhiandri GS, Neilson MM, Lambert RG. Radiation exposure to the sacroiliac joint from low-dose CT compared with radiography. AJR 2018;211:1-5.

23. Jurik AG, Hansen J, Puhakka KB. Effective radiation dose from semicoronal CT of the sacroiliac joints in comparison with axial CT and conventional radiography. Eur Radiol 2002;12:2820-25.

24. Bocker Puhakka K, Jurik AG, Egund N, Schiottz-Christensen B, Stengaard-Pedersen K, van Overeem Hansen G, Vallo Christiansen JV. Imaging of sacroiliitis in early seronegative spondylarthropathy, Assessment of abnormalities by MR in comparison with radiography and CT. Acta Radiologica 2003;44:218-29. 